\title{
artigo
}

Botelho, J.L.S.; Cardoso, F.S.F.; Oliveira, M.D.; Carmo, G.S.; Ruas, R.F.B.; Freitas, I.G.C.;

Cuidado do paciente na atenção primária: Contribuição da tecnologia durante uma pandemia

\section{Cuidado do paciente na atenção primária: Contribuição da tecnologia durante uma pandemia}

\author{
Patient care in primary care: technology's contribution during a pandemic
}

Atención al paciente en atención primaria: contribución de la tecnología durante una pandemia

\section{RESUMO}

Objetivo: Avaliar como e quais tecnologias podem ser implantadas para dar continuidade no cuidado da educação em saúde, tendo enfoque naqueles classificados "grupo de risco" para o COVID-19. Método: Trata- se de uma revisão integrativa realizada nas bases de dados MedLine e Lilacs, no período de 2015 a 2020, ao final realizado uma análise para identificar nos artigos a relevância e elegibilidade. Resultados: Observa - se variadas situações onde foram utilizadas as tecnologias como no rastreio e orientações em teleconsultas, grupos de apoio e amparo á dúvidas em meio virtual a partir das mídias sociais, sites e aplicativos personalizados para repasse de informações sobre sintomas aos usuários. Conclusão: Pode-se constatar a eficiência do uso das tecnologias na atenção primária e com isso, percebe-se uma área inovadora que mostra-se capaz de trazer melhorias para o usuário e facilidade para a equipe de saúde sendo um diferencial para a enfermagem, agregando todas competências em ferramentas informatizadas potencializando a expansão do cuidado.

DESCRITORES: Educação em saúde; Pandemias; Teleatendimento; Continuidade da assistência ao paciente; Betacoronavírus.

\section{ABSTRACT}

Objective: To evaluate how and which technologies can be implemented to continue the care of health education, focusing on those classified as 'risk group' for COVID-19. Method: This is an integrative review carried out in the MedLine and Lilacs databases, from 2015 to 2020, at the end an analysis was carried out to identify the relevance and eligibility in the articles. Results: There are several situations where technologies were used, such as screening and guidance in teleconsultations, support groups and support for doubts in a virtual environment from social media, websites and personalized applications to pass on information about symptoms to users. Conclusion: It can be seen the efficiency of the use of technologies in primary care and with this, an innovative area is perceived that is able to bring improvements for the user and ease for the health team, being a differential for nursing, adding all competences in computerized tools enhancing the expansion of care.

DESCRIPTORS: Health education; Pandemics; Call center; Continuity of patient care; Betacoronavirus.

\section{RESUMEN}

Objetivo: evaluar cómo y qué tecnologías se pueden implementar para continuar la atención de la educación sanitaria, centrándose en los clasificados como 'grupo de riesgo' para COVID-19. Método: Esta es una revisión integradora realizada en las bases de datos MedLine y Lilacs, de 2015 a 2020, al final se realizó un análisis para identificar la relevancia y elegibilidad en los artículos. Resultados: Existen varias situaciones en las que se utilizaron tecnologías, como la detección y orientación en teleconsultas, grupos de apoyo y apoyo a dudas en un entorno virtual desde redes sociales, sitios web y aplicaciones personalizadas para transmitir información sobre los síntomas a los usuarios. Conclusión: se puede observar la eficiencia del uso de tecnologías en la atención primaria y con esto se percibe un área innovadora que puede aportar mejoras para el usuario y facilidad para el equipo de salud, siendo un diferencial para la enfermería, agregando todas las competencias en herramientas computarizadas mejorando la expansión de la atención.

DESCRIPTORES: Educación en salud; Pandemias; Centro de llamadas; Continuidad de la atención al paciente; Betacoronavirus.

RECEBIDO EM: 30/07/2020 APROVADO EM: 05/08/2020

\section{Joyce Lemos de Souza Botelho}

Graduanda em Enfermagem. Faculdades Santo Agostinho - Montes Claros MG.

ORCID: 0000-0002-2284-9844 
Flávio Santiago Ferreira Cardoso

Graduando em Enfermagem. Faculdades Santo Agostinho - Montes Claros MG

ORCID: 0000-0003-4584-2394

\section{Marta Duque de Oliveira}

Graduanda em Enfermagem. Faculdades Santo Agostinho - Montes Claros MG

ORCID: 0000-0002-6567-890X

\section{Gislene Soares do Carmo}

Graduanda em Enfermagem. Faculdades Santo Agostinho - Montes Claros MG.

ORCID: 0000-0002-5217-5991

\section{Rosana Franciele Botelho Ruas}

Enfermeira. Docente do Curso de Enfermagem das Faculdades Santo Agostinho - Montes Claros MG. Mestre em Enfermagem/UFMG. Esp. Gestão Hospitalar e Enfermagem do Trabalho. Doutoranda em Ciências da Saúde - PPGCS/Unimontes.

ORCID: 0000-0002-6649-3966

\section{Ingred Gimenes Cassimiro de Freitas}

Enfermeira - Faculdade de Saúde e Desenvolvimento Humano Santo Agostinho (2012). Mestranda em Cuidado Primário em Saúde - UNIMONTES. Residência de Enfermagem em Saúde da Mulher pelo HUCF- UNIMONTES (2016). Pós-Graduada em Gestão e Auditoria em Saúde - Faculdades Integradas Pitágoras- FIP-MOC (2013). Experiência nas áreas de Saúde da Mulher e do Recém-nascido, Bloco Cirúrgico, Saúde da Família, Saúde do Trabalhador. Docente do curso de enfermagem com função de supervisora de estágio atuando na Estratégia Saúde da Família e Hospitais no município de Montes Claros/MG (Faculdades Santo Agostinho). ORCID: 0000-0001-8325-0985

\section{INTRODUÇÃO}

$\mathbf{N}$ o ano de 2020, ocorreu a inesperada pandemia de SarsCov2 um novo coronavírus, causando a $\mathrm{CO}$ VID-19 por contaminação de pessoa em pessoa ${ }^{1}$. O Brasil enfrenta índices altos de contaminação chegando a 2.419.091 de pessoas positivadas, 87.004 óbitos e 1.634.274 vítimas recuperadas até o dia 27 de julho de $2020^{2}$. Números mundiais mostram que as características sociais e econômicas estão associadas ao nível de contaminação mais agressiva e acentuada que se multiplica, como nos países como Ítalia, Estados Unidos e China ${ }^{4}$.

Várias são as pessoas assintomáticas, no entanto, há pessoas que por suas comorbidades prévias tem o agravamento do estado clínico geral ${ }^{3}$. A enfermagem faz intervenções rotineiramente para manutenção do quadro clínico e prevenção de agravos a pacientes com comorbidades 5 . Além disso, foi adotadas medidas de prevenção como o isolamento social, onde há um distanciamento do profissional e usuário, no entanto, há aqueles que procuram a unidade básica primeiro, mostrando que a população precisa ser assistida ${ }^{4}$.

\section{Várias são}

as pessoas

assintomáticas,

no entanto, há

pessoas que por

suas comorbidades

prévias tem o

agravamento do

estado clínico geral
Umas das formas de se trabalhar a política nacional de promoção da saúde, é através de educações em saúde em que são trabalhados processos temáticos para que a autonomia do cuidado seja expandida pela população $\mathrm{alvo}^{7}$ e dissemine conhecimento para o suporte contínuo do cuidado ${ }^{8}$. Tendo em vista a necessidade do profissional de saúde, pode-se se citar como ferramenta as TICs (tecnologias de Informação e comunicação), adaptadas aos serviços de saúde para suportar os provedores assistenciais e outras como por exemplo, telessaúde ${ }^{6}$. ORIA et al 2018, relata tecnologias como álbum educativo, seriados, cartilhas, vídeos, manuais e oficinas, tendo como suporte o meio telefônico9, além de plataformas sociais que conseguem captar usuários cumprindo com a necessidade estabelecida.

Neste contexto, as tecnologias são ferramentas auxiliadoras na atenção primária. As inovações estudadas trazem novas ideias para implantação de ferramentas em meio a uma esporádica pandemia. $\mathrm{O}$ estudo tem como objetivo avaliar como e quais tecnologias podem ser implantadas para dar continuidade no cuidado, tendo enfoque no "grupo de risco" para o COVID-19. 


\section{artigo}

Botelho, J.L.S.; Cardoso, F.S.F.; Oliveira, M.D.; Carmo, G.S.; Ruas, R.F.B.; Freitas, I.G.C.

Cuidado do paciente na atenção primária: Contribuição da tecnologia durante uma pandemia

\section{MÉTODO}

Trata- se de uma revisão integrativa de literatura. Foram utilizadas as etapas: 1) Escolha da pergunta norteadora; 2) Delimitação dos critérios de inclusão; 3) Representação dos artigos; 4) Análise dos achados; 5) Interpretação de resultados; 6) Descrição das evidências encontradas. Teve como questão norteadora: "Como a tecnologia pode ajudar no cuidado do paciente da atenção pri- mária em meio a uma Pandemia?"

A busca aos artigos científicos aconteceu entre março e julho do ano de 2020, por meio da Biblioteca Virtual em saúde (BVS), nas bases de dados Medical Literature Analyses and Retrieval System Online (MEDLINE) e Literatura Latino-Americana e do Caribe em Ciências da Saúde (LILACS). Para as buscas foram usados os descritores controlados e combinados com operadores booleanos OR e AND. Foram eleitos arti-

Figura 1. Fluxograma de seleção de artigos.

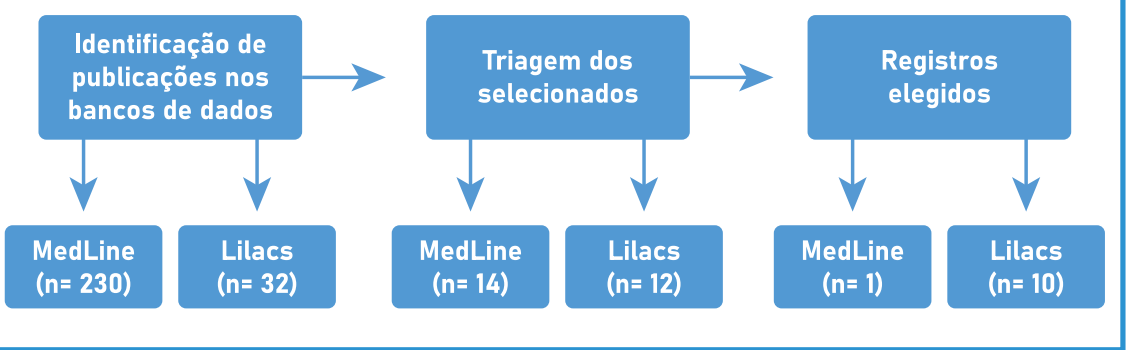

gos disponíveis gratuitamente, em idiomas português e inglês publicados no período de 2015 a 2020. Para a extração de dados, foi elaborado um formulário de equivalentes a características bibliométricas referentes aos artigos selecionados.

\section{RESULTADOS}

Obteve-se como resultado geral nas bases de busca 262 estudos, 32 artigos na LILACS e 230 artigos na MEDLINE. Após a inserção dos filtros, 26 estudos foram potencialmente elegíveis. Seguindo com a leitura completa foram excluídos 15 artigos não adequados, obtendo-se 11 artigos aprovados para amostra final ilustrado na Figura 1.

\section{DISCUSSÃO}

\section{Métodos tecnológicos na educação em saúde}

Estudo realizado nos Estados Unidos,

\section{Quadro 1. Descrição completa das características analisadas nos artigos selecionados}

\begin{tabular}{|c|c|c|c|c|}
\hline ANO E AUTOR & LOCAL & PRÁTICA ABORDADA & REPERCUSSÃO & CATEGORIA IMPLANTADA \\
\hline $\begin{array}{l}\text { Pinto L et al, } 2015 \\
10\end{array}$ & Brasil & Blogs e rede de observatórios & $\begin{array}{l}\text { Aproximação comunicação com a } \\
\text { comunidade }\end{array}$ & Gestão e Educação em Saúde \\
\hline $\begin{array}{l}\text { Ishwaria M et al } \\
201911\end{array}$ & $\begin{array}{l}\text { Estados } \\
\text { Unidos }\end{array}$ & Aplicativo, Mídias sociais & Um bom atendimento & Gestão \\
\hline $\begin{array}{l}\text { Devine K A et al, } \\
201812\end{array}$ & $\begin{array}{l}\text { Estados } \\
\text { Unidos }\end{array}$ & Sites, intervenções telefônicas & Aceitabilidade, promoção da saúde & Educação em saúde \\
\hline $\begin{array}{l}\text { Kang X et al, } 2016 \\
13\end{array}$ & China & Aplicativo de mídia social & Qualidade dos exames e preparos & Educação em saúde \\
\hline $\begin{array}{l}\text { Attai D J et al, } 2015 \\
14\end{array}$ & $\begin{array}{l}\text { Não } \\
\text { especificado }\end{array}$ & Mídias sociais & Envolvimento das mulheres & Educação em saúde \\
\hline $\begin{array}{l}\text { Piscotty R et al, } \\
201515\end{array}$ & $\begin{array}{l}\text { Não } \\
\text { especificado }\end{array}$ & Mídias sociais e smartphones & $\begin{array}{l}\text { Transferência rápida de informa- } \\
\text { ções, melhoria da comunicação }\end{array}$ & Educação em saúde \\
\hline $\begin{array}{l}\text { Chipps J et al, } 2015 \\
16\end{array}$ & África & Telefones Celulares & $\begin{array}{l}\text { Ferramentas importantes utili- } \\
\text { zadas nos celulares, para apren- } \\
\text { dizagem }\end{array}$ & Educação em saúde \\
\hline Ano e autor & Local & Prática abordada & Repercussão & Categoria implantada \\
\hline $\begin{array}{l}\text { Daumas R P et al, } \\
202017\end{array}$ & Brasil & Teleatendimento & $\begin{array}{l}\text { Acompanhamento de agravos, re- } \\
\text { dução de demanda em urgências }\end{array}$ & Gestão e Educação em Saúde \\
\hline $\begin{array}{l}\text { Dimer N A et al, } \\
202018\end{array}$ & Brasil & Telecomunicação e Teleterapia & $\begin{array}{l}\text { Ferramenta satisfatória para as } \\
\text { adversidades }\end{array}$ & $\begin{array}{l}\text { Educação em saúde } \\
\text { Consultas }\end{array}$ \\
\hline $\begin{array}{l}\text { Harzheim E et al, } \\
202019\end{array}$ & Brasil & Teleconsulta, Aplicativo & $\begin{array}{l}\text { Rastreia, monitoria e trata pa- } \\
\text { cientes }\end{array}$ & $\begin{array}{l}\text { Educação em saúde } \\
\text { Consultas }\end{array}$ \\
\hline $\begin{array}{l}\text { Sarti T D et al, } \\
20204\end{array}$ & Brasil & Telessaúde e Telemedicina & $\begin{array}{l}\text { Orientação, rastreio e monitora- } \\
\text { mento de casos da COVID } 19\end{array}$ & $\begin{array}{l}\text { Educação em sáude } \\
\text { Consultas }\end{array}$ \\
\hline
\end{tabular}


com adolescentes e crianças com diagnóstico de câncer, evidenciou recomendações de aplicativos informativos dos sintomas esperados, sistema de avaliação de dor e ainda foram utilizados aplicativos para a análise da aceitação medicamentosa ${ }^{12}$. Resultados positivos também encontrados no uso de aplicativos de mídia social para a instrução correta de preparo no exame de colonoscopia, apresentando números satisfatórios aos anteriores ${ }^{13}$. A partir disto, foi identificado condições em que o próprio profissional conseguisse interagir com o paciente respondendo dúvidas e reforçando todas as orientações médicas necessárias para procedimento ${ }^{6,13}$.

$\mathrm{Na}$ África demonstra que a transferência de informações foi facilitada pelo uso do Smartphone, uma vez que ele estiver conectado a uma rede de internet o usuário ou o profissional consegue acesso a qualquer informação em qualquer local, e se torna possível a criação de fóruns para responder perguntas da população, no entanto se não for utilizada de forma consciente e isolada para tal finalidade acaba se tornando um meio de distração abortando a proposta inicial ${ }^{15,16}$.

As mídias sociais por serem de fácil utilização mostraram grande finalidade como grupos de apoio online, atendendo as demandas surgidas por dúvidas ou quadros de ansiedade ${ }^{14}$. Somado a isto, outro segmento que pode ser implantado são os vídeos temáticos de curta metragem e produções audiovisuais ${ }^{10}$.

\section{Recursos aplicados a consultas}

$\mathrm{O}$ uso do teleatendimento se tornou protocolo nacional de manejo clínico no Brasil, amplia os registros e o acesso á todos na atenção primária, se mostrou eficiente nas teleconsultorias sobre o COVID $19 \mathrm{e}$ sistemas de telemedicina em que os usuários conseguem tirar dúvidas e aprender sobre a prevenção do Sars Cov 2, sem necessariamente ir ao serviço de saúde, e o profissional monitorar os grupos de risco ${ }^{04,19}$.

A partir da telesaúde o paciente têm a integralidade do seu direito á saúde preserva$\mathrm{da}$, o sucesso dessa ferramenta foi tão grande que SARTI et al, 2020, afirma que deveria ser contínuo consultas a distâncias mesmo

fora do contexto de uma pandemia de COVID-19, pois o recurso usado na atenção primária desafogou os serviços de urgência, facilitou o acesso e organizou os fluxos de serviço. No entanto, há um receio da população, em relação a qualidade do atendimento se tornando temerosos com o serviço ${ }^{18}$.

As mídias sociais

por serem de

fácil utilização

mostraram grande

finalidade como

grupos de apoio

online, atendendo

as demandas

surgidas por dúvidas

ou quadros de

ansiedade. Somado

a isto, outro

segmento que pode

ser implantado são

os vídeos temáticos

de curta metragem

e produções

audiovisuais.
Ferramentas para gestão do cuidado

Para DAUMAS et al, 2020, os meios de comunicação como rádios e mídias sociais podem ser utilizados para o repasse de informações com a possibilidade do uso específico para a gestão, acarretando em um serviço mais organizado e ágil para o cliente. Os atendimentos podem ser flexibilizados pelas coletas de informações online, administrar os agendamentos de atendimento presencial para casos de real necessidade, impedindo que o usuário se exponha por exemplo em meio a uma pandemia de COVID-19 de forma desnecessária que poderia ser resolvida por um meio tecnológico em domicílio ${ }^{17,18}$.

Apesar de várias ferramentas serem de cunho simples, os blogs são complexos e exigem certos conhecimentos técnicos, no entanto, vale a pena o uso porque possuem evidências de resultados satisfatórios. Treinamentos podem ser trabalhados para as equipes de saúde desenvolverem habilidades e expandirem seus meios de comunicação e criatividade ${ }^{10}$ principalmente em tempos atípicos de grandes calamidades. As modalidades de meios de comunicação e tecnologia transformaram o contexto educacional da saúde atualmente, onde o impacto foi evidente na gestão dos dados ${ }^{11}$.

\section{CONCLUSÃO}

Com a realização da presente pesquisa pode-se constatar a eficiência do uso das tecnologias de informação e comunicação na atenção primária, não somente em episódios atípicos, como também, existem possibilidades, a partir de protótipos experimentados, que esses meios de comunicação permaneçam presentes nos protocolos oficiais.

Várias são as tecnologias que podem ser utilizadas, algumas exigem maior nível de competência para geri-las, no entanto, nestes casos uma educação continuada 


\section{artigo}

Botelho, J.L.S.; Cardoso, F.S.F.; Oliveira, M.D.; Carmo, G.S.; Ruas, R.F.B.; Freitas, I.G.C.

Cuidado do paciente na atenção primária: Contribuição da tecnologia durante uma pandemia

com a equipe a fim qualificar- los facilita e auxilia a implantação destas.

Com isso, percebe-se uma área inovadora que mostra-se capaz de trazer me- lhorias para o usuário e facilidade para a equipe de saúde, ainda sendo um diferencial para a enfermagem como profissional gestor e cuidador que consegue agregar to- das as suas competências em ferramentas informatizadas potencializando a expansão do cuidado em locais que antes não conseguiam abranger. -

\section{REFERÊNCIAS}

1. BRASIL. Coronavírus (COVID 19) O que é COVID19? Ministério da Saúde. . Brasília, DF, 2020. Acesso em: 20 de julho de 2020. Disponivel em: https://coronavirus.saude.gov.br/sobre-a-doenca\#n-que-e-covid.

2. BRASIL. Painel Coronavírus. Ministério da Saúde. Brasília, DF, 2020. Acesso em: 21 de julho de 2020. Disponivel em: https:// covid.saude.gov.br/.

3. Nedel F. Enfrentando a COVID-19: APS forte agora mais que nunca!. APS [Internet]. 15abr.2020. Disponivel em: <https://apsemrevista.org/aps/article/view/68>. Acesso em 26 de julho de 2020.

4. Sarti, Thiago Dias et al. Qual o papel da Atenção Primária à Saúde diante da pandemia provocada pela COVID-19?. Epidemiologia e Serviços de Saúde [online]. v. 29, n. 2. Disponível em: <https:/doi.org/10.5123/S1679-49742020000200024>. Acesso em 27 de Julho de 2020.

5. TORRES, Ruth Cristini et al. Educação em saúde como ferramenta de enfrentamento das doenças renais crônicas. Journal of Health Connections, Sergipe, Vol. 9, No 2 (2020). Disponivel em: <http://periodicos.estacio.br/index.php/journalhc/article/ viewArticle/6884>. Acesso em 27 de Julho de 2020.

6. CAETANO, Rosângela et al . Desafios e oportunidades para telessaúde em tempos da pandemia pela COVID-19: Uma reflexão sobre os espaços e iniciativas no contexto brasileiro. [Cad. Saúde Pública] v. 36, n. 5, 2020. Disponivel em: $<$ http://www.scielo.br/scielo.php?script=sci_arttext\&pi$\mathrm{d}=\mathrm{S} 0102-311 \times 2020000503001 \& \operatorname{lng}=$ en $\& \mathrm{nrm}=\mathrm{is0}>$. Acesso em 28 de Julho de 2020.

7. FALKENBERG, Mirian Benites et al. Educação em saúde e educação na saúde: conceitos e implicações para a saúde coletiva. [Rev Ciênc. saúde coletiva] Rio de Janeiro, v. 19, n. 3, p. 847-852, Mar. 2014. Disponivel em: < https://doi.org/10.1590/141381232014193.01572013>. Acesso em 27 de Julho de 2020.

8. DOMINGUES, Griesiele Aparecida Silva Ferreira. Experiência do enfermeiro com o uso da tecnologia em cuidados com o paciente com diabetes no pós cirúrgico. [Revista Eletrônica Acervo Saúde] n. 47, p. e3118, 30 abr. 2020. Disponivel em: <https:/doi. org/10.25248/reas.e3118.2020 >. Acesso em 20 de julho de 2020.

9. ORIA, Mônica Oliveira Batista et al. Eficácia de intervenções educativas realizadas por telefone para promoção do aleitamento materno: revisão sistemática da literatura. [Rev. esc. enferm. USP] São Paulo,v.52, 2018. Disponivel em: < https://doi. org/10.1590/s1980-220x2017024303333>. Acesso em 28 de julho de 2020.

10. PINTO, Luiz Felipe. ROCHA, Cristianne Maria Famer. Inovações na Atenção Primária em Saúde: o uso de ferramentas de tecnologia de comunicação e informação para apoio à gestão local. [Ciênc. saúde coletiva online] 2016, vol.21, n.5 pp.1433-1448. Disponivel em: < https://doi.org/10.1590/141381232015215.26662015>. Acesso em: 29 de julho de 2020.

11. Ishwaria M. et al. A Big World Made Small: Using Social Media to Optimize Patient Care. Livro educacional da Sociedade Americana de Oncologia Clínica [revista em internet] 17 de maio de 2019, 39. Disponível em: < https://doi:10.1200/EDBK_246643> Acesso em 30 de julho de 2020.

12. DEVINE, Katie et al. Digital Health Interventions for Adolescent and Young Adult Cancer Survivors. Clinical Cancer Informatics - [ Revista on-line] 29 de junho de 2018. Disponivel em: < https:// doi:10.1200/CCl.17.00138>. Acesso em 30 de julho de 2020.

13. KANG, Xiaoyu et al. Delivery of Instructions via Mobile Social Media App Increases Quality of Bowel Preparation. [Clinical gastroenterology and hepatology: the official clinical practice journal of the American Gastroenterological Association] 14(3), 429-435.e3. 2016. Disponivel em: <https://doi.org/10.1016/j. cgh.2015.09.038>. Acesso em 30 de julho de 2020.

14. ATTAI, Deanna. J et al. Twitter Social Media is an Effective Tool for Breast Cancer Patient Education and Support: Patient-Reported Outcomes by Survey. [Journal of medical Internet research] 17(7), e188. 2015. Disponivel em: <https://doi.org/10.2196/ jmir.4721>. Acesso em: 30 de julho de 2020.

15. PISCOTTY, Ronald et al. Hold the phone? Nurses, social media, and patient care. [Nursing]. 45. 64-67. Mai 2016. Disponivel em: < https://doi: 10.1097/01.NURSE.0000459797.02711.8a. Acesso em 29 de julho de 2020.

16. CHIPPS, Jeniffer et al. Using mobile phones and social media to facilitate education and support for rural-based midwives in South Africa. [Curationis] 38(2), 2015. Disponivel em: <https:// doi.org/10.4102/curationis.v38i2.1500 >. Acesso em: 29 de juIho de 2020 .

17. DAUMAS, Regina Paiva et al. 0 papel da atenção primária na rede de atenção à saúde no Brasil: limites e possibilidades no enfrentamento da COVID-19. [Cadernos de Saúde Pública online]. v. 36, n. 6. Disponivel em: <https://doi.org/10.1590/ 0102-311X00104120>. Acesso em: 28 de julho de 2020.

18. IMER, Nathalia Avila et al. Pandemia do COVID-19 e implementação de telefonoaudiologia para pacientes em domicílio: relato de experiência. [CoDAS online]. 2020, vol.32, n.3. Disponivel em:<https://doi.org/10.1590/2317-1782/20192020144> Acesso em: 29 de junho de 2020.

19. HARZHEIM, Erno et al. Ações federais para apoio e fortalecimento local no combate ao COVID-19: a Atenção Primária à Saúde (APS) no assento do condutor. [Rev. Ciênc. saúde coletiva online].2020,vol.25, pp.2493-2497. Disponivel em: < https://doi. org/10.1590/1413-81232020256.1.11492020>. Acesso em: 30 de junho de 2020. 